\title{
Magnetic stabilization of fluidized beds: Effect of magnetic field orientation
}

\author{
M. J. Espin ${ }^{\mathrm{a}}$, M. A. S. Quintanilla ${ }^{\mathrm{b}}$, J. M. Valverde ${ }^{\mathrm{b}, *}$ \\ ${ }^{a}$ Department of Applied Physics II. University of Seville. Avenida Reina Mercedes s/n, \\ 41012 Sevilla, Spain \\ ${ }^{b}$ Department of Electronics and Electromagnetism. University of Seville. Avenida Reina \\ Mercedes s/n, 41012 Sevilla, Spain
}

\begin{abstract}
Fluidized beds of granular materials can be stabilized by interparticle attractive forces which confer the expanded bed an elastic modulus that stabilizes it against flow perturbations. Stabilization in a structure of enduring contacts is seen to occur naturally due to the universal van der Waals forces for $\sim 50 \mu \mathrm{m}$ particle size beds albeit in a quite reduced interval of gas velocities over the minimum fluidization velocity $v_{\mathrm{mf}}$. As shown in this work, a magnetic field may induce attractive forces between magnetizable particles thus extending the stable fluidization interval well beyond $v_{\mathrm{mf}}$. The structure of the magnetically stabilized bed is however markedly anisotropic since attractive magnetic forces are maximum along the direction of the externally imposed field which leads to the formation of chain particle aggregates. This paper shows experimental measurements on the magnetic yield stress, gas
\end{abstract}

\footnotetext{
*Corresponding author

Email addresses: mjespin@us.es (M. J. Espin), quintani@us.es (M. A. S. Quintanilla), jmillan@us.es (J. M. Valverde)
} 
velocity at the transition to marginal stability and microstructure of magnetostabilized beds as affected by the direction of the magnetic field. Data shows that magnetic stabilization is optimized for co-flow fields as chain aggregates are preferentially orientated parallel to the magnetic field along the direction of minimum drag. As the magnetic field is tilted, particle chains become tilted according to a balance between the magnetic attractive force between the particles and the vertical drag force, which reduces the magnetic yield stress and therefore shortens the interval of magnetic stabilization. Keywords:

Fluidization, Magnetofluidization, Magnetic stabilization, Interparticle forces, Rheology, Magnetorheological fluids

\section{Introduction}

Fluidization is the phenomenon by virtue of which a granular material becomes suspended in a upwards directed fluid flow. This takes place when the superficial fluid velocity $v_{\mathrm{g}}$ reaches a minimum fluidization velocity, $v_{\mathrm{g}}=v_{\mathrm{mf}}$, such that the fluid pressure drop across the particle bed is sufficiently high to support the bed weight per unit area. Gas-solid fluidization technology is widely used in many industrial applications due to the highly efficient contact between solid and gas phases that may be achieved in fluidized bed reactors $[1,2,3,4,5]$.

The behavior of gas-solid fluidized beds depends critically on a variety of physical properties such as particles' size and density, interparticle attractive 
forces and fluid density and viscosity. Geldart [6] proposed a well known four different types of fluidization behavior according to particle size when monodisperse solids are fluidized by dry air at ambient pressure and temperature. Particles of size typically below $d_{p} \simeq 20 \mu \mathrm{m}$ are difficult to fluidize and belong to the Geldart's C group. Strong interparticle attractive forces as compared with particles' weight lead to a cohesive behavior that hinders fluidization. Consequently, these fine powders, when fluidized, tend to rise as a slug of solids or to form channels through which the fluid escapes rather than being homogeneously distributed through the bulk. Different types of techniques have been proposed to help these granular materials being uniformly fluidized such as the use of mechanical stirrers to break up stable channels, the addition of nanosilica beads to coat the solids and increase flowability, or fluidization under high gas pressure and/or with high viscosity gases $[6,7,8,9]$. On the opposite side, Geldart's D group powders, consisting of large-size beads (typically above $d_{p} \gtrsim 500 \mu \mathrm{m}$ ), exhibit a spouting behavior. In an spouted bed, there is a centrally located, upwardly moving diluted core above which the particles drop down to an annular region $[6,10]$. For intermediate size particles, fluidization is characterized by the development of gas bubbles. Materials having a mean particle size between 20 and $100 \mu \mathrm{m}$ appreciably expand beyond $v_{\mathrm{mf}}$ and macroscopic bubbles are not seen until a minimum bubbling velocity $v_{\mathrm{g}}>v_{\mathrm{b}}>v_{\mathrm{mf}}$ is reached. These powders belong to the Geldart's A group. Interparticle attractive forces, which for these powders are similar to particles' weight, may indeed provide 
the fluidized bed with an effective elastic modulus that stabilizes it against small disturbances $[3,11]$. In the stable state $\left(v_{\mathrm{mf}}<v_{\mathrm{g}}<v_{\mathrm{b}}\right)$, interparticle contacts are permanently held by these attractive forces; the bed is jammed and takes the appearance of a weak plastic solid. Jammed bed posses a solid structure consolidated at a low stress and requires a small but non zero yield stress in order to make it flows again (see $[3,11,12,13,14,15]$ for a more detailed description). Powders of typical size above $d_{p} \gtrsim 100 \mu \mathrm{m}$ belong to Geldart's B group [6]. Geldart's B fluidized beds are commonly unstable, being characterized by the development of large gas bubbles rising across the bed just at the onset of fluidization $\left(v_{\mathrm{b}} \approx v_{\mathrm{mf}}\right)$. Bubbling may limit the performance of Geldart's B fluidized bed reactors due to the bypassing of gas through bubbles, which reduces gas-solid contact effectiveness and leads to uncertainties in process scale up [16].

Artificial enhancement of interparticle forces may suppress the growth of gas bubbles and shift the behavior of fluidized beds from Geldart B to Geldart A. For example, bubbling beds can be stabilized by incremental addition of a liquid [17], by fluidizing them with highly adsorbing gases [18] or by fluidization at high temperature and pressure conditions [3, 7]. However, fluidization at high temperatures, the addition of liquids or the use of gases distinct to air may lead to material sintering, attrition [19], or adsorption/desorption of chemical species [20], which would modify particle properties and cause undesired fluidization behavior of the powder as well. An alternative noninvasive method for enhancing interparticle forces to stabilize bubbling beds 
consists of the application of external magnetic fields [21, 22]. For magnetizable particles, sufficiently strong magnetic fields cause mechanical structuring of particles $[23,24]$ which significantly affect heat and mass transfer properties as well as the rheological behavior of these so-called magnetofluidized beds (MFBs). Several studies have demonstrated that the application of a magnetic field to Geldart's B magnetic granular materials yields bubbling suppression beyond the minimum fluidization velocity. Channels and bubbles could be also eliminated by imposing magnetic fields to Geldart's group C magnetic powders [25]. In the case of Geldart's group A magnetic powders the range of gas velocities at which the fluidized bed is stabilized is extended when external magnetic fields are applied [15, 26, 27].

Magnetically stabilized beds (MSBs) are useful for a variety of potential applications such as the adsorption of contaminants from a gas stream. Magnetic stabilization has also been studied for the development of magnetically controlled valves $[28,29,30]$. In addition, a better understanding of the rheological properties of magnetized particles is of interest in geological flows [31] and magnetorheological fluids (MRFs) [32]. The interested reader may find additional details on the history and current state of the art of MFBs in extended reviews reported elsewhere [33, 34, 35, 36, 37]. Histrov has recently published a series of extended reviewes focused on the application of magnetic fields as assisting fluidization technique [38, 39, 40, 41, 42, 43, 44, 45, 46].

In the present work we have investigated the magnetofluidization behavior of a magnetite powder, which belongs to the class of Geldart A powders. Our 
main goal is to study the effect of the orientation of the magnetic field on the structure and on the mechanical strength of the MSB.

\section{Materials and experimental setup}

\subsection{Materials}

The magnetic powder used in our work consists of nearly spherical and monodisperse magnetite particles manufactured and supplied to us by Xerox Co (Fig. 1). A detailed material characterization is described in [26, 27, 47].

Particle density is $\rho_{p}=5060 \mathrm{~kg} / \mathrm{m}^{3}$, as measured by means of an AccuPyc 1330 pycnometer. A Morphologi G3S (Malvern) characterization device was used to obtain an average particle size $d_{\mathrm{p}}=26.2 \mu \mathrm{m}$ (determined from the circular equivalent diameter), elongation $e_{\mathrm{p}}=0.098$ (one minus the particle aspect ratio), and circularity $c_{\mathrm{p}}=0.938$ (ratio of the particle's projected area to the square of its perimeter).

A magnetic analysis of the powder was performed by two different procedures reported in detail elsewere $[15,26,47]$. In the range of field intensities applied in our experiments $(H<5 \mathrm{kA} / \mathrm{m})$, a superconducting quantum interference device (SQUID) magnetometer (SQUID Quantum Design MPMS XL) showed that the initially demagnetized particles exhibit a superparamagnetic behavior characterized by a linear and reversible response to the applied magnetic field intensity. A complementary method was used to obtain the magnetic susceptibility of the particles by measuring the change in self-inductance $L$ of a single layer solenoid as the magnetite powder was 
poured into it. The material (particle) susceptibility was estimated to be $\chi_{\mathrm{p}}=11.5$ from the coherent potential approximation (CPA) mixing rule equation [48].

\subsection{Experimental setup}

The setup used in our work (Fig. 2) is an adaptation of the Sevilla Powder Tester (SPT) to perform magnetofluidization experiments (see [15] and [47] for a detailed description). In this apparatus, the powder bed sample is held in a vertically oriented cylindrical vessel made of polycarbonate $(2 R=2.6 \mathrm{~cm}$ internal diameter in the tests reported in this paper) and rests on a nonmagnetizable porous plate ( $5 \mu \mathrm{m}$ pore size) which acts as gas distributor. Air is used as fluidizing gas after being passed across a set of filters and a Refrigerated Air Dryer (model SMC IDFA3E) to remove any possible polluting particles and humidity. By means of a series of computer-controlled valves and a mass flow controller (MKS model 1179A, $2000 \mathrm{~cm}^{3} / \mathrm{min}$ full scale), a flow of the filtered and dried air is pumped through the powder bed while the gas pressure drop $\Delta p$ across it is measured by a differential pressure transducer (MKS model 220CD, 10 Torr full scale). The height $h$ of the bed, which provides an average value of the particle volume fraction $\phi$, is measured by means of an ultrasonic sensor (Senix model Ultra-S) placed on top of the vessel.

Magnetite powders have been tested as affected by uniform magnetic fields applied along different directions by placing the bed in the center of 
a pair of square Helmholtz coils $(50 \mathrm{~cm} \times 50 \mathrm{~cm})$ that can be rotated. The orientation of the applied magnetic field was determined from the angle $\theta$ between the field and the vertical direction (see Fig. 2). The intensity $H_{\mathrm{c}}$ of the externally imposed magnetic field was controlled by adjusting the electrical current supplied by a N8741A Agilent current source circulating through the coils. Measurements taken by a magnetic probe (Hirst Magnetics Gaussmeter) show that the magnetic field strength is uniform throughout the volume occupied by the powder bed.

\section{Experimental results}

Figure 3 illustrates experimental results on the evolution of the gas pressure drop across the bed (normalized by the powder weight per unit area) $\Delta p / W$ as the superficial gas velocity $v_{\mathrm{g}}$ is increased in a typical magnetofluidization test. The first step of the experiment consists of applying a magnetic field after the sample is subjected to a sufficiently large gas flow to reach the bubbling regime $\left(v_{\mathrm{g}}>v_{\mathrm{b}}\right)$. Under these conditions, $\Delta p$ is roughly equal to $W$ since the whole structure of the bed is sustained by the gas flow. The bed remains in this fluid-like bubbling regime until $v_{\mathrm{g}}$ is slowly reduced to a critical value $v_{\mathrm{J}}$ known as the jamming velocity. A further decrease of $v_{\mathrm{g}}$ below $v_{\mathrm{J}}$ causes the bed to jam and it transits to a solid-like stable state. Note that in this manuscript (as well as in our previos works devoted to MFBs) the term jamming means the evolution from a bubbling bed to an expanded but fixed bed in part sustained by the gas flow and in part by the endur- 
ing interparticle contacts. A detail description of the different order-disorder transitions between fixed beds, MSBs and MFBs and the usual terms used to named them can be found in [38]. This transition can be identified in Fig. 3 by the falling of $\Delta p$ below $W$. Part of the weight becomes then sustained by the enduring network of interparticle contacts in the stabilized bed, which gives rise to a consolidation stress $\sigma_{\mathrm{c}}=W-\Delta p\left(v_{\mathrm{g}}\right)$ at the bottom layer of the powder. For each test, a minimun gas velocity $v_{0}$ was set at which the consolidation stress reaches a value $\sigma_{0}=\sigma_{\text {cmax }}=W-\Delta p\left(v_{0}\right)$. According to the Janssen equation [49], friction at the walls does not support a significant fraction of the powder weight if the height of the bed is below the bed diameter as is the case in our experiments.

In order to measure the effect of the applied magnetic field on the mechanical strength of the MSB, the gas velocity was then progressively increased from $v_{0}$ while the external magnetic field is kept fixed. This is called the H-ON or MAGNETIZATION LAST operation mode $[47,50,51,52]$ in contrast with the H-OFF-ON or MAGNETIZATION FIRST operation mode where the applied magnetic field is imposed once the bed is settled at a gas velocity $v_{0}$ (the interested reader may check [53] for further details on the effect of the magnetic field orientation on fixed bed). For small values of $v_{\mathrm{g}}$, the solid-like structure remains unperturbed (bed height does not change) and $\Delta p$ linearly increases with $v_{\mathrm{g}}$. The slope $s$ of this linear relationship between $\Delta p$ and $v_{\mathrm{g}}$ is indicated in Fig. 3. At the value of $v_{\mathrm{g}}$ at which $\Delta p$ equals $W$ (the minimum fluidization velocity, $v_{\mathrm{mf}}$ ), the bed should transit again to the fluid-like 
state. However, for cohesive materials, as magnetized powders, permanent interparticle contacts can be still held by the attractive forces between the particles. In Fig. 3, this feature is shown by the increase of $\Delta p$ over $W$ which indicates the existence of a tensile strength of the bed. As the gas velocity is further increased, the tension builds up to a point at which the powder breaks, bed height expansion is initiated and the pressure drop falls down to around $W$. The tensile yield stress $\sigma_{\mathrm{t}}$ required to break the powder can be thus obtained as the maximum value of $\Delta p-W$, which takes place just before the breaking of the bed. This condition is met first at the bottom of the bed, where the tensile stress is larger and a fracture surface is first observed. Further increase of $v_{\mathrm{g}}$ beyond the breaking point causes propagation of the fracture to the rest of the bed and the eventual transition to a bubbling regime. The fluidization cycle described above was repeated for different values of $v_{0}$ and for applied magnetic fields of different intensities $H_{\mathrm{c}}$ and tilt angles $\theta$ with respect to the vertical direction.

To explore the effect of the applied magnetic field on the stabilization and fluidization of the magnetite powder, the jamming velocity $v_{\mathrm{J}}$, the rate of increase $s$ of $\Delta p$ vs. $v_{\mathrm{g}}$ and the tensile yield stress $\sigma_{\mathrm{t}}$ will be analyzed. Data on the gas velocity at the jamming transition $v_{\mathrm{J}}$ are plotted in Fig. 4 as a function of the intensity $H_{\mathrm{c}}$ and the tilt angle $\theta$ of the applied magnetic field. As may be observed, the range of gas velocities at which the MFB is stabilized $\left(v_{0}<v_{\mathrm{g}}<v_{\mathrm{J}}\right)$ is extended with the field intensity. For $H_{\mathrm{c}} \gtrsim 1.5-2.0 \mathrm{kA} / \mathrm{m}$, the increase of $v_{\mathrm{J}}$ with $H_{\mathrm{c}}$ becomes significantly marked. On the other hand, 
at a given value of $H_{\mathrm{c}}$, the MFB is jammed at higher gas velocities as the external field becomes more aligned with the gravity field. These trends with $H_{\mathrm{c}}$ and $\theta$ agree with those reported by Histrov on MSBs under the MAGNETIZATION FIRST operation mode [53]. As expected, there is no effect of $v_{0}$ on $v_{\mathrm{J}}$ since initialization by bubbling erases any memory of the previous consolidated structure of the MSB.

Figure 5 illustrates the effect of the magnetic field on the slope $s$ between $\Delta p$ and $v_{\mathrm{g}}$ before the fracture takes place. This parameter is inversely proportional to the bed permeability to the gas flow according to Darcy's law for the passage of a fluid through a porous solid at low Reynolds numbers [54]. In the absence of an external field, it is observed that $s$ decreases with $v_{0}$. This can be explained by the reduction of the consolidation stress $\sigma_{0}$ as $v_{0}$ increases which yields higher porosity $(\epsilon=1-\phi)$ and therefore a lower gas pressure drop across the bed for a given superficial gas velocity above $v_{0}$. When an external magnetic field is imposed to the powder bed, an additional reduction of $s$ is observed, which is particularly relevant for $H_{\mathrm{c}}$ above $1.5-2.0 \mathrm{kA} / \mathrm{m}$. Larger $H_{\mathrm{c}}$ thus give rise to more permeable MSBs to the gas flow. As regards to the orientation of the applied field, Fig. 5 shows that the rate of decrease of $s$ with $H_{\mathrm{c}}$ is more marked as $\theta$ is decreased. Thus, more vertically orientated magnetic fields also hinders the resistance of the bed to the passing of gas flow. The dependence of $s$ on $H_{\mathrm{c}}$ is strongly affected by the consolidation velocity $v_{0}$ at which the bed is magnetically stabilized. As $v_{0}$ is increased, the variation of $s$ with $H_{\mathrm{c}}$ and $\theta$ becomes less pronounced. 
Figure 6 shows the measured tensile yield stress of the MSBs as a function of the intensity and tilt angle of the imposed magnetic field. As previously observed in Figs. 4 and 5 for $v_{\mathrm{J}}$ and $s$, there is a limited effect of the external fields for intensities below $1.5-2.0 \mathrm{kA} / \mathrm{m}$. For larger $H_{\mathrm{c}}$, measured values of $\sigma_{\mathrm{t}}$ significantly increase as compared to those obtained in the absence of an external field. The application of magnetic fields with lower tilt angles also contributes to further enhance of $\sigma_{\mathrm{t}}$ as the magnetic field becomes more parallel to the gas flow and the gravity field. Finally, it is seen that $\sigma_{\mathrm{t}}$ decreases as MSBs are subjected to lower consolidation stresses.

\section{Discussion}

\subsection{The naturally stabilized bed}

The rheology of fluidized beds is critically influenced by the balance of forces acting on the particles. In the absence of external fields that might induce interparticle forces and for Geldart B powders, fluidization takes place when the Stockes drag force due to the gas flow overcomes the particles weight at $v_{\mathrm{mf}}$. As $v_{\mathrm{g}}$ is decreased from the minimum bubbling velocity, there comes a point at which the strength of the drag force is decreased below the particles weight and the bed jams at $v_{\mathrm{J}}$. When the powder is composed of cohesive particles, the drag force must overcome as well the interparticle cohesive forces in order to fluidize the bed. In the absence of humidity and external fields, the interparticle attractive force is mainly due to the universal van der Waals force that arises from the interaction of fluctuating molecular electric 
dipole fields. If retardation effects are negligible and the interaction between molecules is considered as pairwise, the van der Waals force can be calculated as $[55]$

$$
f_{\mathrm{vdW}} \simeq \frac{A d^{*}}{12 z_{0}^{2}}
$$

where $A$ is the Hamaker constant, $z_{0}$ is the distance of closest approach between two molecules and $d^{*}$ is the size of the surface asperities at interparticle contact. Typical values reported in literature for these parameters are $A \simeq 10^{-19} \mathrm{~J}[56,57], z_{0} \simeq 3-4 \AA$ and $d^{*} \simeq 0.2 \mu \mathrm{m}$ [3]. Thus, the van der Waals force can be estimated as $f_{\mathrm{vdW}} \approx 10 \mathrm{nN}$. In order to determine the degree of cohesiveness of a powder, the attractive interparticle force is usually compared with particle weight by means of the granular Bond number $B o_{\mathrm{g}}=3 A d^{*} /\left(20 \pi \rho_{\mathrm{p}} g d_{\mathrm{p}}^{3} z_{0}^{2}\right)[58]$. For the magnetite particles used in our work, $B o_{\mathrm{g}} \sim 2$ indicating that it is only a slightly cohesive powder (as expected for a Geldart A material). However, the equation 1 is only valid for unloaded particles. When powder beds are settled at $v_{0}<<v_{\mathrm{J}}$, the consolidation stress $\sigma_{0}$ induces plastic deformation of asperities at contacts. Including the effect of plastic yielding on the interparticle adhesion force requires more elaborated expressions than Eq. 1 taking into account the mechanical properties of the solids [58], which is beyond the scope of this manuscript (interested readers may see $[59,60,58]$ for a detailed review). Figure 7 shows the average particle volume fraction $\phi_{0}$ of the stabilized beds derived from direct measurements 
of the bed height at different values of $v_{0}, H_{\mathrm{c}}$ and $\theta$. For decreasing consolidation stresses $\sigma_{0}$, powder beds stabilize at more open structures (smaller $\phi_{0}$ ) with fewer interparticle contacts and less prone to suffer plastic deformation [61]. This justifies the reduction of $\sigma_{\mathrm{t}}$ with $v_{0}$ as observed in Fig. 6 as has been extensively reported for other cohesive powders $[13,58]$. Moreover, as the structure of the stabilized bed becomes more expanded with increasing $v_{0}$, the resistance to the passage of the gas flow is reduced, which explains the decrease of the initial slope $s$ in Fig. 5 .

\subsection{The magnetostabilized bed}

When an external magnetic field is applied to a fluidized bed of magnetizable particles in the bubbling regime, particles rearrange into chained aggregates due the induced attractive forces [15, 47]. If such particles are made of a linear magnetizable material of relative permeability $\mu_{\mathrm{p}}$ much larger than the relative permeability of the fluidizing gas $\mu_{\mathrm{f}}$, as occurs for magnetite and air in this work $\left(\mu_{\mathrm{p}}=1+\chi_{\mathrm{p}}=12.5 \gg \mu_{\mathrm{f}} \approx 1\right)$, the magnetic interparticle force $\vec{f}_{\mathrm{m}}$ can be estimated from a multipolar expansion. Let us consider two spherical particles of an isolated linear chain aggregate (Fig. 8) whose centers are separated by a distance $r$. The magnetic force between these particles can be calculated as [62, 63]:

$$
\vec{f}_{\mathrm{m}}=f_{\mathrm{mr}} \hat{e}_{\mathrm{r}}+f_{\mathrm{m} \alpha} \hat{e}_{\alpha}=f_{\mathrm{m}}^{0}\left(\frac{d_{\mathrm{p}}}{r}\right)^{4}\left[\left(2 f_{\|} \cos ^{2} \alpha-f_{\perp} \sin ^{2} \alpha\right) \hat{e}_{\mathrm{r}}+\left(f_{\Gamma} \sin (2 \alpha)\right) \hat{e}_{\alpha}\right],
$$


where $f_{\mathrm{m}}^{0}=3 / 16 \pi \mu_{0} d_{\mathrm{p}}^{2}\left[\left(\mu_{\mathrm{p}}-\mu_{\mathrm{f}}\right) /\left(\mu_{\mathrm{p}}+2 \mu_{\mathrm{f}}\right)\right]^{2} H^{2}, \mu_{0}$ is the vacuum permeability, $H$ is the intensity of the imposed magnetic field $\left(H=H_{\mathrm{c}}\right.$, in this work) and $\alpha$ is the angle formed between the line joining the centers of the two spheres and the direction of the external field (Fig. 8). The terms $f_{\|}, f_{\perp}$ and $f_{\Gamma}$ are the force coefficients and depend on the magnetic permeabilities and the distance between particles. Using the algorithm developed in [63], we have calculated these coefficients and it is observed that, at a given $\mu_{\mathrm{p}}$, $f_{\|}$, and $f_{\Gamma}$ decrease with $r$ in contrast to $f_{\perp}$, which remains roughly constant [27]. Moreover, $f_{\|}$is always significantly larger than $f_{\perp}$ and $f_{\Gamma}$ regardless of the distance between particles. For instance, for a MSB of magnetite beads $\left(\mu_{\mathrm{p}}=12.5\right)$, and for contacting particles $\left(r \approx d_{\mathrm{p}}\right)$, the values of these coefficients are $f_{\|}=9.585, f_{\perp}=0.607$ and $f_{\Gamma}=1.581[47,27]$. Thus, the radial attractive component of the force predominates over the tangential and repulsive components which leads to a net attractive force even for relatively large values of $\alpha$ (Fig. 8).

In order to analyze the measured rheological properties of MFBs described in the above section, the balance between the gas shear force and the magnetic force as well as the changes in the microstructure of the bed caused by these forces must be considered. The frictional force due to the fluidizing gas tends to align the chains of particles in the vertical direction. By contrast, the magnetic interaction promotes the formation of such chains in the direction of the imposed field along which the attractive force is maximum. Data in Figure 7 illustrates the modification of the MSB structure that results 
from this competition. In this figure, measured values of $\phi_{0}$ are plotted as a function of $H_{\mathrm{c}}, \theta$ and $v_{0}$. As can be observed, the bed stabilizes at more expanded structures as the intensity of the field is increased and/or the field is more vertically orientated (decreasing $\theta$ ). Expansion becomes progressively less marked as the gas velocity at which the bed stabilizes $v_{0}$ is increased, particularly for $H_{\mathrm{c}}$ below $2.0 \mathrm{kA} / \mathrm{m}$. At a given $H_{\mathrm{c}}$, as more horizontally oriented magnetic fields are applied to the sample (increasing $\theta$ ), the shear forces progressively increase the angle $\alpha$ between the chained particles and the field direction which reduces the strength of $f_{\mathrm{m}}$ according to Eq. 2. Thus, the shear force due to the gas flow is able to balance the induced magnetic force at lower $v_{\mathrm{J}}$ (Fig. 4) where the bed is more compacted (larger $\phi_{0}$ in Fig. 7). Under these circumstances, the resistance to the passing of the gas flow across the MSB is increased as shown by the increase of $s$ in Fig. 5 .

If now $\theta$ is fixed and $H_{\mathrm{c}}$ is increased, stronger magnetic interactions reduce the misalignment produced by the drag force between the chained aggregates and the field direction. This fact promotes the strength of the chain of particles, which requires more intense shear forces to be broken. The transition from the bubbling to the stabilized regime takes then place at larger $v_{\mathrm{J}}$ (as seen in Fig. 4) and smaller $\phi_{0}$ (Fig. 7). A larger porosity of these MSBs favors the passing of the gas flow, which is reflected in a reduction of $s$ in Fig. 5 .

Finally, the mechanical strength of the MSB when an external mag- 
netic field is applied will be discussed. The measured tensile yield stress $\sigma_{\mathrm{t}}=\sigma_{\mathrm{t}}\left(H_{\mathrm{c}}, \theta, v_{0}\right)$ in Fig. 6 stems from two different contributions. On one hand, we have the natural yield stress $\sigma_{\mathrm{t}}\left(H_{\mathrm{c}}=0, v_{0}\right)$ arising from the van der Waals interaction and plastic yielding of particle contacts at a given consolidation velocity $v_{0}$ (data at $H_{\mathrm{c}}=0$ in Fig. 6 ). On the other hand, we have the magnetic yield stress $\sigma_{\mathrm{mt}}$ caused by the interparticle magnetic forces induced by the external magnetic field. Figure 9 shows the values of this magnetic contribution to the measured overall tensile yield strength, which is obtained as $\sigma_{\mathrm{mt}}=\sigma_{\mathrm{t}}\left(H_{\mathrm{c}}, \theta, v_{0}\right)-\sigma_{\mathrm{t}}\left(H_{\mathrm{c}}=0, v_{0}\right)$ (see [26] for more details about the procedure to estimate $\left.\sigma_{\mathrm{mt}}\right)$. First of all, it is worth mentioning that, within the experimental accuracy, $\sigma_{\mathrm{mt}}$ only depends on $H_{\mathrm{c}}$ and $\theta$ indicating that the increase of number of contacts per particle as $v_{0}$ is decreased is not significant. Note also that $\sigma_{\mathrm{mt}}$ is larger than $\sigma_{\mathrm{t}}\left(H_{\mathrm{c}}=0, v_{0}\right)$ even for the lowest intensities of the applied magnetic field. This could be explained by the comparison of the magnitude of van deer Waals and the magnetic forces between particles. Of the several possible definitions of the magnetic Bond number in fluidized beds [42], we chose the ratio of the typical value of the attractive component of the magnetic force between particles (Eq. 2) to the particle weight $B o_{\mathrm{g}}=12 f_{\|} f_{0} /\left(\pi \rho_{\mathrm{p}} g d_{\mathrm{p}}^{3}\right)$. For $H_{\mathrm{c}} \approx 0.5 \mathrm{kA} / \mathrm{m}$, this dimensionless number is $B o_{\mathrm{g}} \sim 4$ which is larger than the typical value of the non-magnetic Bond number $\left(B o_{\mathrm{g}} \sim 2\right)$. On the other hand, the rheology of MSBs is affected by the orientation of the applied field for $H_{\mathrm{c}}$ above $1.5 \mathrm{kA} / \mathrm{m}$. Indeed, the effect of the field in a MFB would be expected to be noticeable when $B o_{\mathrm{g}} \gtrsim 10$ 
[64] which occurs at $H_{\mathrm{c}} \approx 1.0 \mathrm{kA} / \mathrm{m}$ for the previous definition of the magnetic bond number. This feature is also in agreement with the changes of the microstructure of MSBs illustrated in Fig. 7. The compaction of MSBs is reduced as $f_{\mathrm{m}}$ becomes more intense. Indeed, at a given $H_{\mathrm{c}}$ and as $\theta$ is decreased, more expanded MSBs can be stabilized due to the existence of larger $\sigma_{\mathrm{mt}}$. On the other hand, at a fixed orientation of the applied field, the mechanical strength (larger $\sigma_{\mathrm{mt}}$ ) and the porosity of the microstructure of MSBs are promoted as $H_{\mathrm{c}}$ increases.

\subsection{Tilt angle of chained aggregates}

To conclude this work, we will look for a relationship between the interparticle magnetic force $\vec{f}_{\mathrm{m}}$ and the structuring of MSBs. It must be pointed out that the magnetic field appearing in Eq. 2 should be the magnetic field in the bulk of the sample $H_{\mathrm{b}}$ which is the sum of the externally applied field $H_{\mathrm{c}}$ (created by the Helmholtz coils) plus the field induced by the magnetized powder (also known as the demagnetizing field) $H_{\mathrm{d}}$. For a linearly magnetizable material (as is the case of the magnetite powder used in our work), $H_{\mathrm{b}}$ can be obtained from the bulk magnetization $M$ of the bed and the bulk demagnetization factor $\kappa$ as:

$$
H_{\mathrm{b}}=H_{\mathrm{c}}+H_{\mathrm{d}}=H_{\mathrm{c}}-\kappa M=\frac{H_{\mathrm{c}}}{1+\kappa \chi_{\mathrm{b}}}
$$

where $\chi_{\mathrm{b}}=M / H_{\mathrm{b}}$ is the bulk magnetic susceptibility of the sample. 
Using the coherent potential approximation (CPA) mixing rule equation $[48,47,27], \chi_{\mathrm{b}}$ can be derived from the particle volume fraction $\phi$ and the magnetic susceptibility of the material $\chi_{\mathrm{p}}$ :

$$
\chi_{\mathrm{b}}=\chi_{\mathrm{p}} \phi \frac{3+4 \chi_{\mathrm{b}}}{3+\chi_{\mathrm{p}}+3 \chi_{\mathrm{b}}} .
$$

Let us approximate the magnetite powder to a solid cylinder of the same dimensions (height and diameter) and magnetic susceptibility $\chi_{b}$ than the MSB subjected to an external magnetic field tilted an angle $\theta$. According to the procedure detailed in $[65,66]$, a finite element method (FEM) commercial program (COMSOL) was used to calculate the demagnetization factors in the radial $\kappa_{x, \theta}$ and vertical $\kappa_{z, \theta}$ directions using the bulk magnetic susceptibility of the cylinder and the cylinder aspect ratio [65]. Since $\chi_{\mathrm{b}}$ depends on $\chi_{\mathrm{p}}$ and $\phi$, Eq. 4 was employed to obtain both demagnetization factors as functions of the particle volume fraction of the MSB. Figure 10 shows the dependence of $\kappa_{x, \theta}$ and $\kappa_{z, \theta}$ on $\phi$ for different tilt angles $\theta$. Lines corresponds to the best fittings of the results to a logarithmic law $\kappa=a+\ln \phi$ where the best fitting parameters $a$ and $b$ are reported in Table 1 . At given values of $H_{\mathrm{c}}, \theta$ and $\phi$, the radial $H_{\mathrm{bx}}$ and vertical $H_{\mathrm{bz}}$ components of the magnetic field inside the bulk (and hence the overall $H_{\mathrm{b}}=\sqrt{H_{\mathrm{bx}}^{2}+H_{\mathrm{bz}}^{2}}$ ) could be obtained from the corresponding values $\kappa_{x, \theta}$ and $\kappa_{z, \theta}$ as:

$$
H_{\mathrm{bx}}=\frac{H_{\mathrm{c}} \cos \theta}{1+\kappa_{x, \theta} \chi_{\mathrm{b}}}
$$




$$
H_{\mathrm{bz}}=\frac{H_{\mathrm{c}} \sin \theta}{1+\kappa_{z, \theta} \chi_{\mathrm{b}}}
$$

Theoretical models for MSBs and MRFs [39, 66, 67, 68] predict that the magnetic contribution to the yield stress should scale as $\sigma_{\mathrm{mt}} \sim \xi \phi f_{\mathrm{m}}$ where $\xi$ is the average number of contacts per particle (coordination number). Since the strength of the MSB is due to the radial component of $f_{\mathrm{m}}$ in Eq. 2 and $f_{\|}$is much larger than $f_{\perp}$, the attractive magnetic force can be roughly approximated as $f_{\mathrm{mr}} \sim H_{\mathrm{b}}^{2} \cos ^{2} \alpha$. Then $\sigma_{\mathrm{mt}}$ can be estimated as:

$$
\sigma_{\mathrm{mt}}=K \xi \phi H_{\mathrm{b}}^{2} \cos ^{2} \alpha
$$

where $K$ depends on the magnetic properties and size of the particles as well as the characteristic constants of each particular theoretical model. Figure 11(b) shows an image of the bulk of the magnetite bed stabilized by a vertically oriented magnetic field $\left(\theta=0^{\circ}\right)$. As observed, particles aggregate into chains aligned with the external applied field $\left(\alpha=0^{\circ}\right.$ in Eq. 2 and Fig. 8). Using the equation $\xi=1.61(1-\phi)^{-1.48}$ to determine the coordination number [69] and Eq. 7, $K=\sigma_{\mathrm{mt}} /\left(\xi \phi H_{\mathrm{b}}^{2} \cos ^{2} \alpha\right)$ where $\sigma_{\mathrm{mt}}, \phi_{0}$ and $H_{\mathrm{b}}$ should be now replaced by their corresponding values at $\theta=0^{\circ}$ (and $\alpha=0^{\circ}$ ). This parameter allows us to obtain $\alpha$ from Eq. 7 for other orientations of the external magnetic field. Figure 12 shows data of the estimated angle $\beta=\theta-\alpha$ between chain aggregates and the vertical direction (Fig. 8) vs. $H_{\mathrm{b}}$ and $\theta$. As expected, with the exception of $\theta \neq 0^{\circ}$ (Fig. 11(b)), chains of particles 
are not aligned with the applied magnetic field $(\beta \neq \theta)$ due to the shear force of the fluidizing gas. This result is consistent with direct visualization of the chain aggregates in the bulk of the MSB for $\theta=90^{\circ}$ in Fig. 11(c). Snapshots were acquired by a binocular magnifying lens coupled to a CCD camera (the arrangement of the Helmholtz coils in the experimental set up did not allow us to take pictures at the other orientations tested $-\theta=30^{\circ}$ or $60^{\circ}$ - without modifying the microstructure of the MSB). The tilt angle directly measured from these images is $\beta \approx 40^{\circ}$ showing a reasonably agreement with the angle obtained from Eq. 7. Note also that chain aggregates become less tilted (smaller $\beta$ ) as $H_{\mathrm{b}}$ is increased. The same trend can be obtained by imposing more vertically orientated (smaller $\theta$ ) magnetic fields. Histrov reported a similar trend on the inclination of the free surface of MSBs subjected to magnetic fields with different orientations [39]. These data also agree with the above reported measurements of $\phi_{0}$ in Fig. 7. The strength of chain aggregates (as determined by $f_{\mathrm{m}}$ in Eq. 2) is promoted as $H_{\mathrm{c}}$ is increased and/or $\theta$ is decreased. This allows chains to keep their integrity as they become more vertically oriented (smaller $\beta$ ) by increasing gas velocities $v_{\mathrm{g}}$ which gives rise to looser (smaller $\phi_{0}$ ) microstructures of the MSB.

Although data in Fig. 12 serve to qualitatively describe the changes in the microstructure of MSBs induced by magnetic fields of different orientations, a more complex analysis would be required in a future work. For instance, Eq. 7 overestimates the attractive component $f_{\mathrm{mr}}$ of the magnetic force between particles (the repulsive radial component is neglected). In addition, Eq. 
2 does not include the effects off the surrounding particles on the magnetic interaction between magnetizable particles $[70,71,72]$. On the other hand, all usual expressions reported in literature [73] to compute $\xi$ from $\phi$ (including that used in this work) are derived for random assemblies of equal sized spheres while the microstructure of MSBs is formed by clusters of chained of particles aggregated in a preferential direction.

\section{Conclusions}

This work has analyzed the effect of an externally imposed magnetic field on the stabilization of a fluidized bed of magnetite particles. Due to naturally occurring van der Waals forces of attraction between these particles (of similar order than particle weight), the fluidized bed is stabilized albeit just for a short interval of gas velocities beyond the minimum fluidization velocity over which part of the bed weight is sustained by enduring network of interparticle contacts. The imposition of a magnetic field serves to further extend the stable fluidization interval depending on the strength and orientation of the field as shown in our work. Induced attractive forces between magnetized particles lead to the formation of chain aggregates preferentially orientated along the field direction. On the other hand, the gas drag force favors orientation of these chains along the vertical direction. Thus, magnetic stabilization is optimized for vertical magnetic fields. Experimental measurements reported in this work show that the stable fluidization interval is shortened whereas the magnetic yield stress and bed expansion are reduced 
as the magnetic field is tilted. Chain aggregates are seen then inclined at an angle that depends on the interplay of the magnetic attractive force between the particles and the gas drag force. Thus, magnetic stabilization may be fine-tuned non-invasively by means of the intensity and orientation of the externally imposed magnetic field.

\section{Acknowledgements}

This work was supported by Andalusian Government (Junta de Andalucia contract No. FQM-5735) and Spanish Goverment Agency Ministerio de Economia y Competitividad (contract No. CTQ2014-52763-C2-2-R and FIS2014-54539-P). The Microscopy, Functional Characterization, and X-ray services of the Innovation, Technology and Research Center of the University of Seville (CITIUS) are gratefully acknowledged.

\section{References}

[1] D. Kunii, O. Levenspiel, Fluidization Engineering, ButterworthHeinemann, 1991.

[2] M. Rhodes, Introduction to Particle Technology, 2nd Edition, Wiley, 2008. doi:10.1002/9780470727102.

[3] K. Rietema, The Dynamics of Fine Powders, Springer Netherlands, 1991. doi:10.1007/978-94-011-3672-3. 
[4] F. A. Zenz, Fluidization Phenomena And Fluidized Bed Technology, in: Fayed, L. Otten (Eds.), Handb. Powder Sci. Technol., Springer US, 1997, pp. 487-531.

[5] J. Zhu, Fluidization of fine powders, in: S. J. Antony, W. Hoyle, Y. Ding (Eds.), Granul. Mater., Royal Society of Chemistry, Cambridge, 2004, pp. 270-295. doi:10.1039/9781847550996.

[6] D. Geldart, Types of gas fluidization, Powder Technol. 7 (5) (1973) 285292. doi:10.1016/0032-5910(73)80037-3.

[7] M. Kwauk, J. Li, D. Liu, Particulate and aggregative fluidization - 50 years in retrospect, Powder Technol. 111 (1-2) (2000) 3-18. doi:http://dx.doi.org/10.1016/S0032-5910(00)00234-5.

[8] J. Li, J. Kuipers, Effect of pressure on gassolid flow behavior in dense gas-fluidized beds: a discrete particle simulation study, Powder Technol. 127 (2) (2002) 173-184. doi:http://dx.doi.org/10.1016/S00325910(02)00116-X.

[9] J. M. Valverde, Fluidization of Fine Powders: Cohesive versus Dynamical Aggregation, Springer Netherlands, 2013.

[10] D. Gidaspow, Multiphase Flow and Fluidization: Continuum and Kinetic Theory Descriptions, Academic Press, 1994.

[11] R. Jackson, The Dynamics of Fluidized Particles, Cambridge University Press, 2000. 
[12] S. C. Tsinontides, R. Jackson, The mechanics of gas fluidized beds with an interval of stable fluidization, Journal of Fluid Mechanics 255 (1993) 237-274. doi:10.1017/S0022112093002472.

[13] P. K. Watson, J. M. Valverde, A. Castellanos, The tensile strength and free volume of cohesive powders compressed by gas flow, Powder Technol. 115 (1) (2001) 45-50.

[14] S. Sundaresan, Instabilities in fluidized beds, Annu. Rev. Fluid Mech. 35 (1) (2003) 63-88. doi:10.1146/annurev.fluid.35.101101.161151.

[15] M. J. Espin, J. M. Valverde, M. A. S. Quintanilla, A. Castellanos, Rheology of magnetofluidized fine powders: The role of interparticle contact forces, J. Rheol. 54 (4) (2010) 719-734. doi:10.1122/1.3380851.

[16] J. P. Constantineau, J. R. Grace, C. J. Lim, G. G. Richards, Generalized bubbling-slugging fluidized bed reactor model, Chem. Eng. Sci. 62 (1-2) (2007) 70-81. doi:10.1016/j.ces.2006.08.011.

[17] J. Seville, R. Clift, The effect of thin liquid layers on fluidisation characteristics, Powder Technol. 37 (1) (1984) 117-129. doi:10.1016/00325910(84)80011-X.

[18] H.-Y. Xie, D. Geldart, Fluidization of FCC powders in the bubble-free regime: effect of types of gases and temperature, Powder Technol. 82 (3) (1995) 269-277. doi:http://dx.doi.org/10.1016/0032-5910(94)02932-E. 
[19] F. Scala, F. Montagnaro, P. Salatino, Attrition of Limestone by Impact Loading in Fluidized Beds, Energy Fuels 21 (5) (2007) 2566-2572. doi:10.1021/ef0700580.

[20] J. Blamey, E. Anthony, J. Wang, P. Fennell, The calcium looping cycle for large-scale \{CO2\} capture, Prog. Energ. Combust. Sci. 36 (2) (2010) 260-279. doi:http://dx.doi.org/10.1016/j.pecs.2009.10.001.

[21] T. W. Johnson, J. R. Melcher, Electromechanics of Electrofluidized Beds, Ind. Eng. Chem. Fundam. 14 (3) (1975) 146-153. doi:10.1021/i160055a002.

[22] M. V. Filippov, The effect of a magnetic field on a ferromagnetic particle suspension bed, Prikl. Magnitogidrodin. Tr. Inst. Fiz. Akad. Nauk. Latv. SSR 12 (1960) 215-236.

[23] J. Casal, J. Arnaldos, The structure of magnetized-fluidized beds, Powder Technol. 64 (1) (1991) 43 - 48. doi:http://dx.doi.org/10.1016/00325910(91)80004-3.

[24] W. Resnick, Y. Zimmels, D. Boadi, Magnetic structural effects of flow through beds of magnetizable particles, IEEE Trans. Magn. 24 (2) (1988) 757-760. doi:10.1109/20.11335.

[25] Q. Zhu, H. Li, Study on magnetic fluidization of group C powders, Powder Technol. 86 (2) (1996) 179-185. doi:http://dx.doi.org/10.1016/00325910(96)83162-7. 
[26] M. J. Espin, J. M. Valverde, M. A. S. Quintanilla, Stabilization of fluidized beds of particles magnetized by an external field: effects of particle size and field orientation, J. Fluid Mech. 732 (2013) 282-303. doi:10.1017/jfm.2013.403.

[27] M. J. Espin, M. A. S. Quintanilla, J. M. Valverde, Effect of particle size polydispersity on the yield stress of magnetofluidized beds as depending on the magnetic field orientation , Chem. Eng. J. 277 (2015) 269-285. doi:http://dx.doi.org/10.1016/j.cej.2015.04.124.

[28] V. Ivanova, J. Hristov, E. Dobreva, Z. Al-Hassan, I. Penchev, Performance of a magnetically stabilized bed reactor with immobilized yeast cells, Appl. Biochem. Biotechnol. 59 (2) (1996) 187-198. doi:10.1007/BF02787820.

[29] C. Webb, H.-K. Kang, G. Moffat, R. A. Williams, A.-M. Estévez, J. Cuéllar, E. Jaraiz, M.-A. Galán, The magnetically stabilized fluidized bed bioreactor: a tool for improved mass transfer in immobilized enzyme systems?, Chem. Eng. J. Bioch. Eng. J. 61 (3) (1996) 241-246. doi:10.1016/0923-0467(95)03043-3.

[30] A. Cohen, T. Chi, Aerosol filtration in a magnetically stabilized fluidized bed, Powder Technol. 64 (1-2) (1991) 147-158. doi:10.1016/00325910(91)80015-B.

[31] D. K. Potter, A. Stephenson, Field-induced magnetic anisotropy in a 
dilute dispersion of gamma Fe2O3 particles, J. Appl. Phys. 63 (5) (1988) 1691-1693. doi:http://dx.doi.org/10.1063/1.339903.

[32] J. de Vicente, D. J. Klingenberg, R. Hidalgo-Alvarez, Magnetorheological fluids: a review, Soft Matter 7 (8) (2011) 3701-3710. doi:10.1039/c0sm01221a.

[33] G. M. Colver, The influence of electric and magnetic fields on airfluidized beds, in: H. Littman (Ed.), Proc. NSF Work. Fluid. FluidParticle Syst. Res. Needs Priorities Work. Held Rensselaer Polytech. Institute, Troy, New York 12181, Oct. 17, 18, 19, 1979, National Science Foundation (U.S.). Department of Chemical Engineering and Environmental Engineering, Rensselaer Polytechnic Institute, Washington DC, 1979, p. 57.

[34] W.-K. Lee, A review of the rheology of magnetically stabilized fluidized beds, Powder Technol. 64 (1-2) (1991) 69-80. doi:10.1016/00325910(91)80007-6.

[35] Y. Liu, R. Hamby, R. D. Colberg, Fundamental and practical developments of magnetofluidized beds: A review, Powder Technol. 64 (1-2) (1991) 3-41. doi:10.1016/0032-5910(91)80003-2.

[36] J. Siegell, Early studies of magnetized-fluidized beds, Powder Technol. 57 (3) (1989) 213-220. doi:10.1016/0032-5910(89)80077-4. 
[37] R. L. Sonolikar, Magneto-fluidized Beds, in: L. K. Doraiswamy, A. S. Mujumdar (Eds.), Transp. Fluid. Part. Syst., Elsevier, 1989, pp. 359423.

[38] J. Hristov, Magnetic Field Assisted Fluidization - A Unified Approach Part 1. Fundamentals and relevant hydrodynamics of gas-fluidized beds (batch solids mode), Rev. Chem. Eng. 18 (4-5) (2002) 295-512. doi:10.1515/REVCE.2002.18.4-5.295.

[39] J. Hristov, Magnetic Field Assisted Fluidization - A Unified Approach Part 2. Solids Batch Gas-Fluidized Beds: Versions and Rheology., Rev. Chem. Eng. 19 (1) (2003) 1-132. doi:10.1515/REVCE.2003.19.1.1.

[40] J. Hristov, Magnetic Field Assisted Fluidization - A Unified Approach Part 3: Heat Transfer in Gas-Solid Fluidized Beds - a critical reevaluation of the results, Rev. Chem. Eng. 19 (3) (2003) 229-355. doi:10.1515/REVCE.2003.19.3.229.

[41] J. Hristov, Magnetic Field Assisted Fluidization - A Unified Approach Part 4. Moving Gas-Fluidized Beds, Rev. Chem. Eng. 20 (5-6) (2004) 377-550. doi:10.1515/REVCE.2004.20.5-6.377.

[42] J. Hristov, Magnetic Field Assisted Fluidization - A Unified Approach Part 5. A hydrodynamic treatise on liquid-solid fluidized beds, Rev. Chem. Eng. 22 (4-5) (2006) 195-375. doi:10.1515/REVCE.2006.22.45.195 . 
[43] J. Hristov, Magnetic Field Assisted Fluidization - A Unified Approach Part 6. Topics of Gas-Liquid-Solid Fluidized Bed Hydrodynamics, Rev. Chem. Eng. 23 (6) (2007) 373-526. doi:10.1515/REVCE.2007.23.6.373.

[44] J. Hristov, Magnetic Field Assisted Fluidization - A Unified Approach Part 7. Mass Transfer: Chemical reactors, basic studies and practical implementations thereof, Rev. Chem. Eng. 25 (1-2-3) (2009) 1-254. doi:10.1515/REVCE.2009.25.1-2-3.1.

[45] J. Hristov, Magnetic Field Assisted Fluidization - A Unified Approach Part 8. Mass transfer: magnetically assisted bioprocesses, Rev. Chem. Eng. 26 (3-4) (2010) 55-128. doi:10.1515/REVCE.2010.006.

[46] J. Hristov, Magnetic Field Assisted Fluidization - A Unified Approach Part 9. Mechanical processing with emphasis on separations, Rev. Chem. Eng. 28 (4-6) (2012) 243-308. doi:10.1515/revce-2012-0015.

[47] M. J. Espin, J. M. Valverde, M. A. S. Quintanilla, A. Castellanos, Stabilization of gas-fluidized beds of magnetic powders by a cross-flow magnetic field, J. Fluid Mech. 680 (2011) 80-113. doi:10.1017/jfm.2011.151.

[48] K. Karkkainen, A. Sihvola, K. Nikoskinen, Analysis of a threedimensional dielectric mixture with finite difference method, IEEE Trans. Geosci. Remote Sens. 39 (5) (2001) 1013-1018. doi:10.1109/36.921419. 
[49] R. M. Nedderman, Statics and Kinematics of Granular Materials, Cambridge University Press, Cambridge, 1992. doi:10.1017/CBO9780511600043.

[50] J. Siegell, Liquid-fluidized magnetically stabilized beds, Powder Technology 52 (2) (1987) 139 - 148. doi:http://dx.doi.org/10.1016/00325910(87)80144-4.

[51] J. Y. Hristov, Fluidization of ferromagnetic particles in a magnetic field part 2: Field effects on preliminarily gas fluidized bed, Powder Technology 97 (1) (1998) 35 - 44. doi:http://dx.doi.org/10.1016/S00325910(97)03392-5.

[52] J. M. Valverde, M. J. Espin, M. A. S. Quintanilla, A. Castellanos, Magnetofluidization of fine magnetite powder, Phys. Rev. E 79 (2009) 031306. doi:10.1103/PhysRevE.79.031306.

[53] J. Y. Hristov, Fluidization of ferromagnetic particles in a magnetic field part 1: The effect of field line orientation on bed stability, Powder Technology 87 (1) (1996) $59-66$.

[54] P. Carman, Fluid Flow Through Granular Beds, Trans. - Inst. Chem. Eng. 15 (1937) $150-166$.

[55] H. Hamaker, The London-van der Waals attraction between spherical particles, Physica 4 (10) (1937) 1058-1072. 
[56] J. Visser, On Hamaker constants: A comparison between Hamaker constants and Lifshitz-van der Waals constants, Advances in Colloid and Interface Science 3 (4) (1972) 331-363.

[57] R. J. Hunter, Colloidal systems and interfaces, by s. ross and i. d. morrison. 1st ed. (1988) pp. xviii + 422. john wiley and son, new york., The Canadian Journal of Chemical Engineering 67 (1) (1989) 175-176. doi:10.1002/cjce.5450670128.

[58] A. Castellanos, The relationship between attractive interparticle forces and bulk behaviour in dry and uncharged fine powders, Adv. Phys. 54 (4) (2005) 263-376. doi:10.1080/17461390500402657.

[59] K. L. Johnson, Contact Mechanics, Cambridge University Press, 1985, cambridge Books Online. doi:http://dx.doi.org/10.1017/CBO9781139171731.

[60] S. D. Mesarovic, N. A. Fleck, Frictionless indentation of dissimilar elastic-plastic spheres, Int. J. Solids and Struct. 37 (46-47) (2000) 70717091.

[61] M. Suzuki, K. Makino, M. Yamada, K. Iinoya, A Study on the Coordination Number in a System of Randomly Packed, Uniform-Sized Spherical Particles, Int. Chem. Eng. 21 (1981) 482-488.

[62] D. J. Klingenberg, F. van Swol, C. F. Zukoski, The small shear rate response of electrorheological suspensions. I. Simulation in 
the point-dipole limit, J. Chem. Phys. 94 (9) (1991) 6160-6169. doi:10.1063/1.460402.

[63] H. J. H. Clercx, G. Bossis, Many-body electrostatic interactions in electrorheological fluids, Phys. Rev. E 48 (4) (1993) 2721-2738. doi:10.1103/PhysRevE.48.2721.

[64] J. M. Valverde, A. Castellanos, Types of gas fluidization of cohesive granular materials, Phys. Rev. E 75 (2007) 031306. doi:10.1103/PhysRevE.75.031306.

[65] D.-X. Chen, E. Pardo, A. Sanchez, Fluxmetric and magnetometric demagnetizing factors for cylinders, J. Magn. Magn. Mater. 306 (1) (2006) 135-146. doi:10.1016/j.jmmm.2006.02.235.

[66] M. A. S. Quintanilla, M. J. Espin, J. M. Valverde, Effect of magnetic field orientation on fluidized beds of magnetic particles: Theory and experiment, Particuology 12 (2014) 54-63. doi:10.1016/j.partic.2013.03.002.

[67] G. Bossis, O. Volkova, S. Lacis, A. Meunier, Magnetorheology: Fluids, Structures and Rheology, in: S. Odenbach (Ed.), Ferrofluids Magn. Control. Fluids Their Appl., Vol. 594 of Lecture Notes in Physics, Springer Berlin Heidelberg, Berlin, Heidelberg, 2002, pp. 202-230. doi:10.1007/3540-45646-5.

[68] N. M. Wereley, Magnetorheology: Advances and Applications, Royal Society of Chemistry, 2013. 
[69] M. Nakagaki, H. Sunada, Theoretical studies on structures of the sedimentation bed of spherical particles, Yakugaku Zasshi 88 (6) (1968) $651-5$.

[70] R. A. Anderson, Electrostatic Forces in an Ideal SphericalParticle Electrorheological Fluid, Langmuir 10 (9) (1994) 2917-2928. doi:10.1021/la00021a013.

[71] Y. Chen, A. F. Sprecher, H. Conrad, Electrostatic particle-particle interactions in electrorheological fluids, J. Appl. Phys. 70 (11) (1991) 67966803. doi:10.1063/1.349855.

[72] R. Tao, Q. Jiang, Simulation of structure formation in an electrorheological fluid, Phys. Rev. Lett. 73 (1) (1994) 205-208. doi:10.1103/PhysRevLett.73.205.

[73] M. Suzuki, Packing Properties, in: Masuda, K. Higashitani, H. Yoshida (Eds.), Powder Technol. Fundam. Part. Powder Beds, Part. Gener., CRC Press, 2006, pp. 293-308. 
Table 1: Best fitting parameters of the calculated demagnetization factor in the radial $\kappa_{x, \theta}$ and vertical $\kappa_{z, \theta}$ directions $[65,66]$ to the expression $\kappa=a+b \ln \phi$. The fitting correlation factor is $\rho^{2}$.

\begin{tabular}{|c|c|c|c|c|c|c|}
\hline & \multicolumn{3}{|c|}{$\kappa_{x, \theta}$} & \multicolumn{3}{c|}{$\kappa_{z, \theta}$} \\
\hline$\theta\left(^{\circ}\right)$ & $a$ & $b$ & $\rho^{2}$ & $a$ & $b$ & $\rho^{2}$ \\
\hline 0 & - & - & - & 0.0381 & -0.5494 & 0.9991 \\
\hline 30 & 0.1155 & -0.158 & 0.9974 & 0.033 & -0.4758 & 0.9991 \\
\hline 60 & 0.2001 & -0.2737 & 0.9974 & 0.0191 & -0.2747 & 0.9991 \\
\hline 90 & 0.2311 & -0.316 & 0.9974 & - & - & - \\
\hline
\end{tabular}



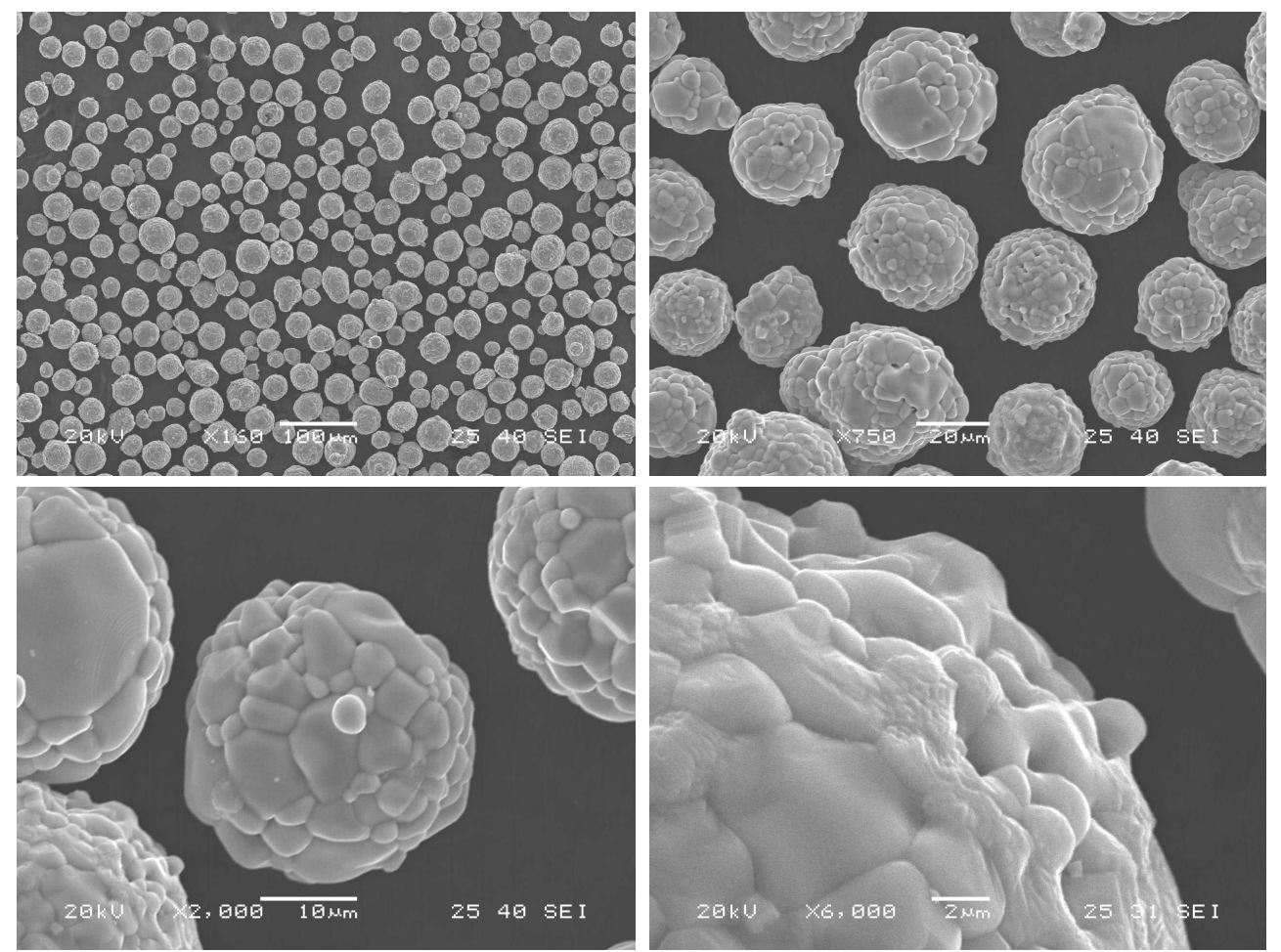

Figure 1: Scanning electron microscopy (SEM) photographs of the magnetite particles used in the magnetofluidization experiments reported in this paper.
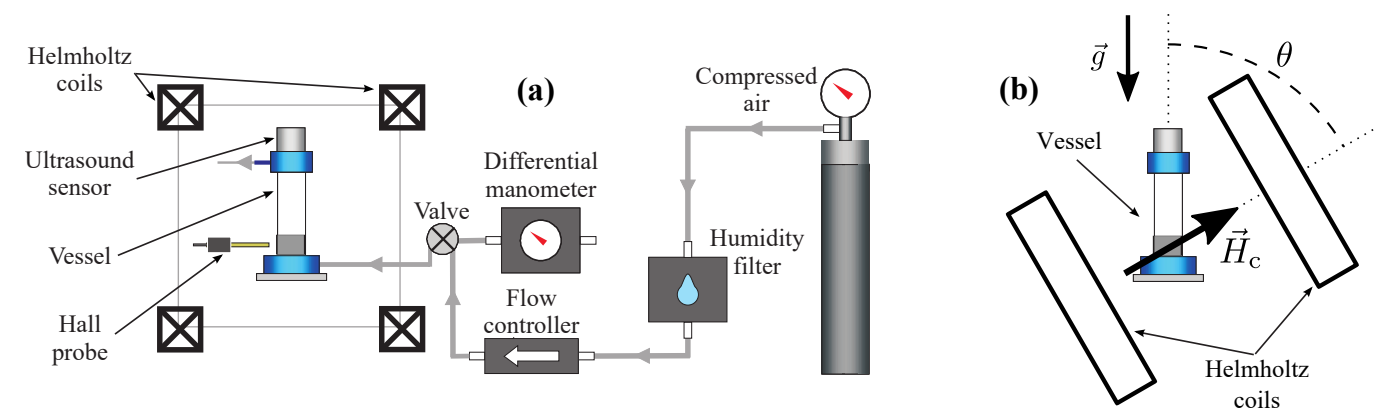

Figure 2: Sketch of the experimental setup used in the magnetofluidization experiments reported in this work (a). Tilt angle $\theta$ of the applied external magnetic field $\vec{H}_{\mathrm{c}}$ with respect the vertical direction (b). 


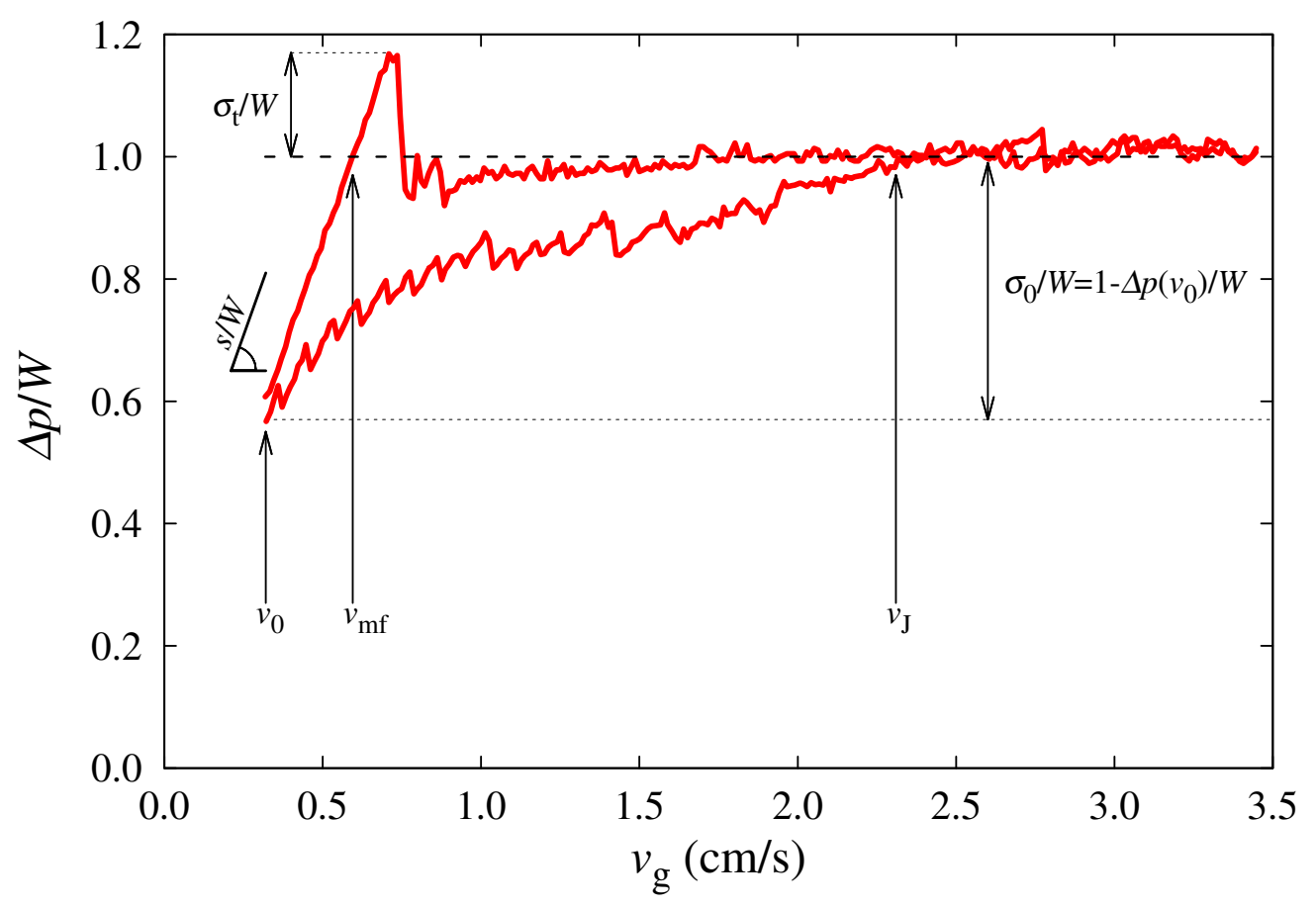

Figure 3: Example of a typical fluidization-defluidization cycle obtained in the magnetofluidization experiments. Data of the measured gas pressure drop across the bed $\Delta p$ (normalized by the powder weight per unit area $W$ ) as a function of the superficial gas velocity $v_{\mathrm{g}}$. The labels indicate the gas velocity at the jamming transition $v_{\mathrm{J}}$; the gas velocity $v_{0}$ at which the bed is consolidated at a maximum stress $\sigma_{0}=W-\Delta p\left(v_{0}\right)$; the initial slope $s$ between $\Delta p$ and $v_{\mathrm{g}}$ (before the fracture of the bed); the minimum fluidization velocity $v_{\mathrm{fm}}$ and the measured tensile yield stress of the bed $\sigma_{\mathrm{t}}$. In this test, the powder sample was consolidated at a gas velocity $v_{0}=0.320 \mathrm{~cm} / \mathrm{s}$ and subjected to an external magnetic field of intensity $H_{c}=2.89 \mathrm{kA} / \mathrm{m}$ tilted $\theta=60^{\circ}$ with respect to gravity. 


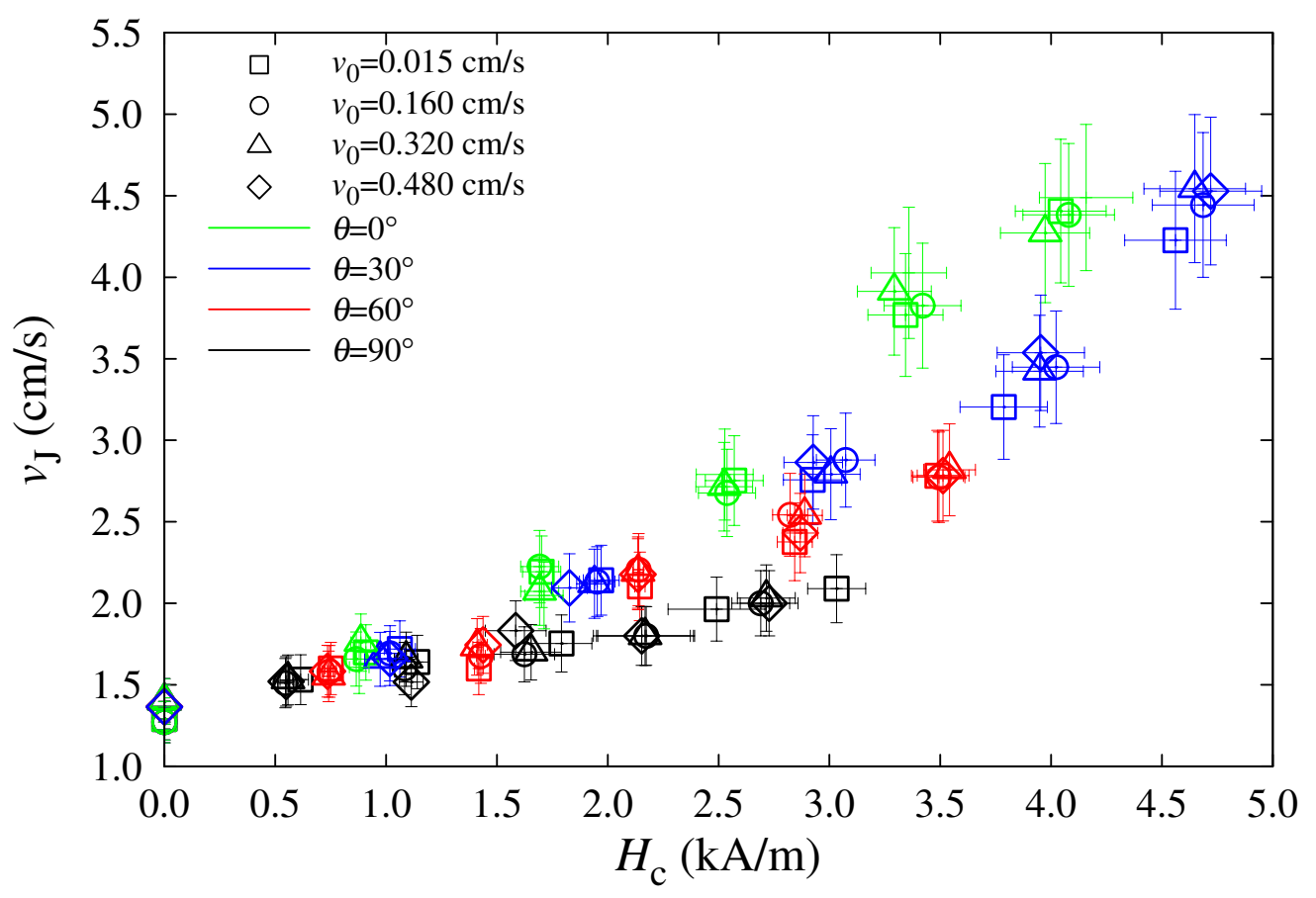

Figure 4: Gas velocity at the jamming transition $v_{\mathrm{J}}$ versus the magnetic field intensity $H_{\mathrm{c}}$ for different values of the tilt angle $\theta$ of the applied field with respect to the vertical direction. Consolidation velocities $v_{0}$ are indicated. 

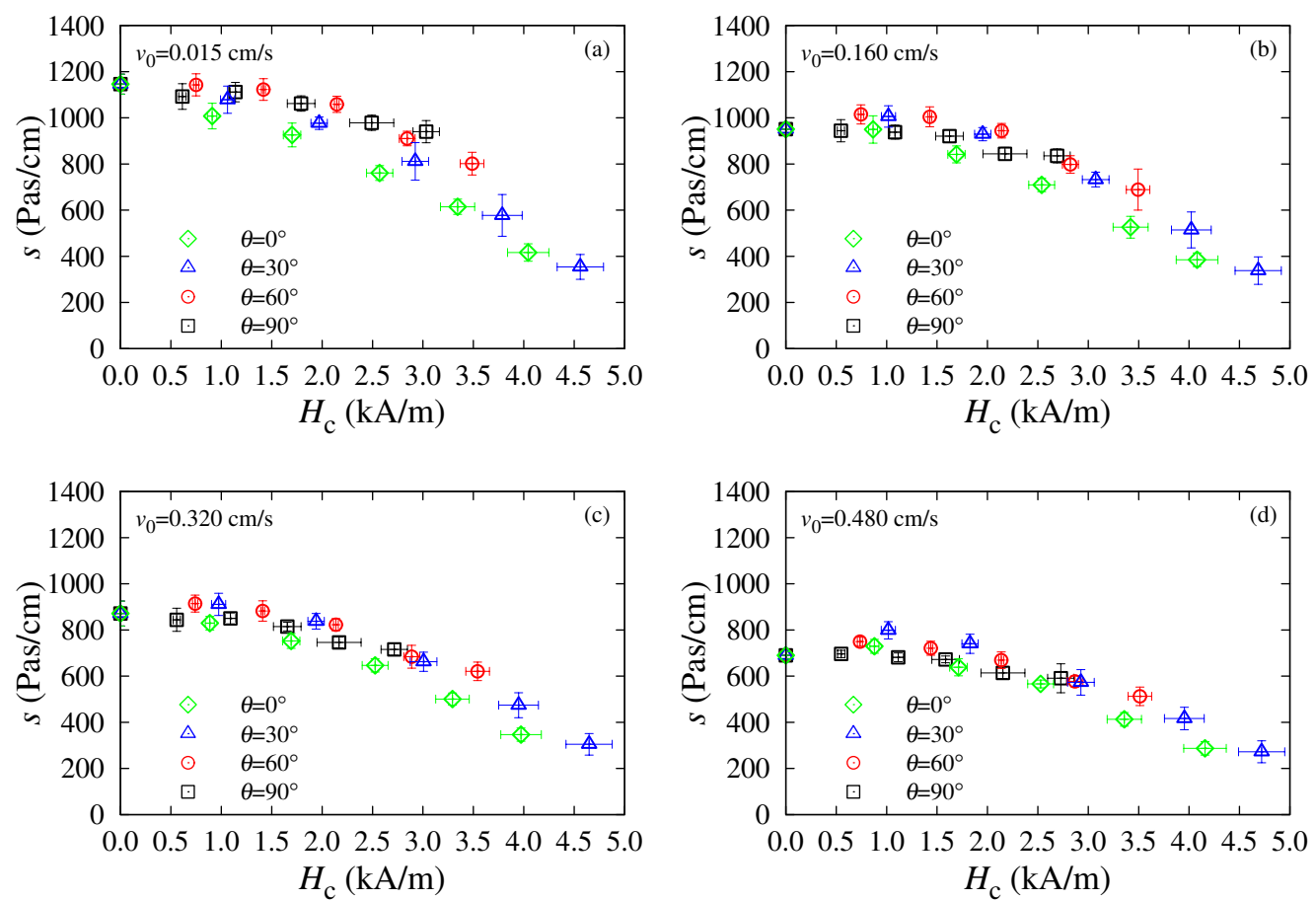

Figure 5: Slope of the gas pressure drop $\Delta p$ vs. the gas velocity $v_{\mathrm{g}}$ before the breaking point as a function of the intensity $H_{\mathrm{c}}$ and tilt angle $\theta$ of the externally applied magnetic field. Data obtained for $v_{0}=0.015 \mathrm{~cm} / \mathrm{s}(\mathrm{a}), v_{0}=0.160 \mathrm{~cm} / \mathrm{s}(\mathrm{b}), v_{0}=0.320 \mathrm{~cm} / \mathrm{s}(\mathrm{c})$ and $v_{0}=0.480 \mathrm{~cm} / \mathrm{s}(\mathrm{d})$, respectively. 

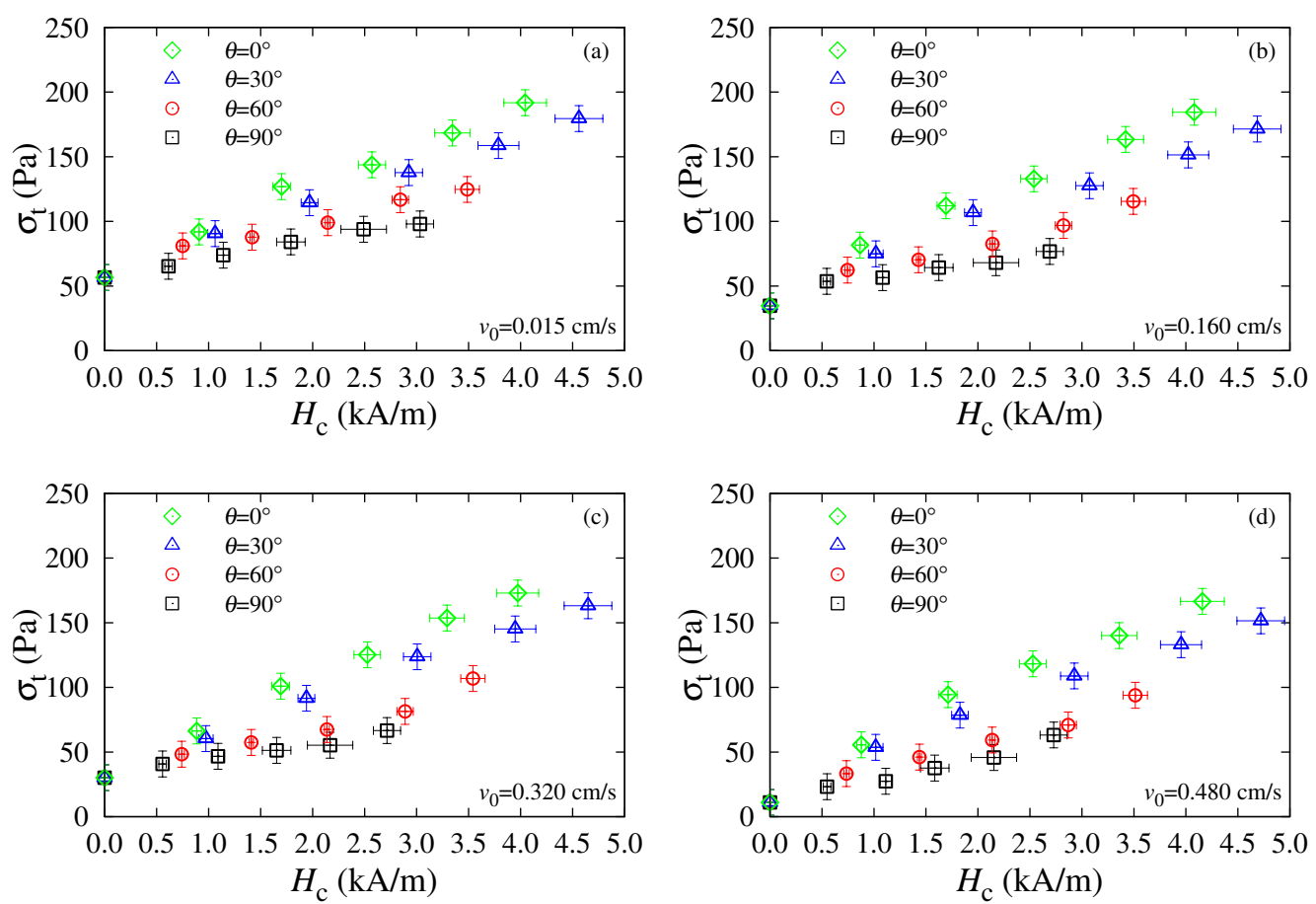

Figure 6: Tensile yield stress $\sigma_{\mathrm{t}}$ of the MSBs as a function of the intensity $H_{\mathrm{c}}$ of the external magnetic field applied at the indicated tilt angles with respect to the vertical direction. Experimental data were obtained at different consolidation velocities $v_{0}=0.015 \mathrm{~cm} / \mathrm{s}(\mathrm{a})$, $v_{0}=0.160 \mathrm{~cm} / \mathrm{s}(\mathrm{b}), v_{0}=0.320 \mathrm{~cm} / \mathrm{s}(\mathrm{c})$ and $v_{0}=0.480 \mathrm{~cm} / \mathrm{s}(\mathrm{d})$. 

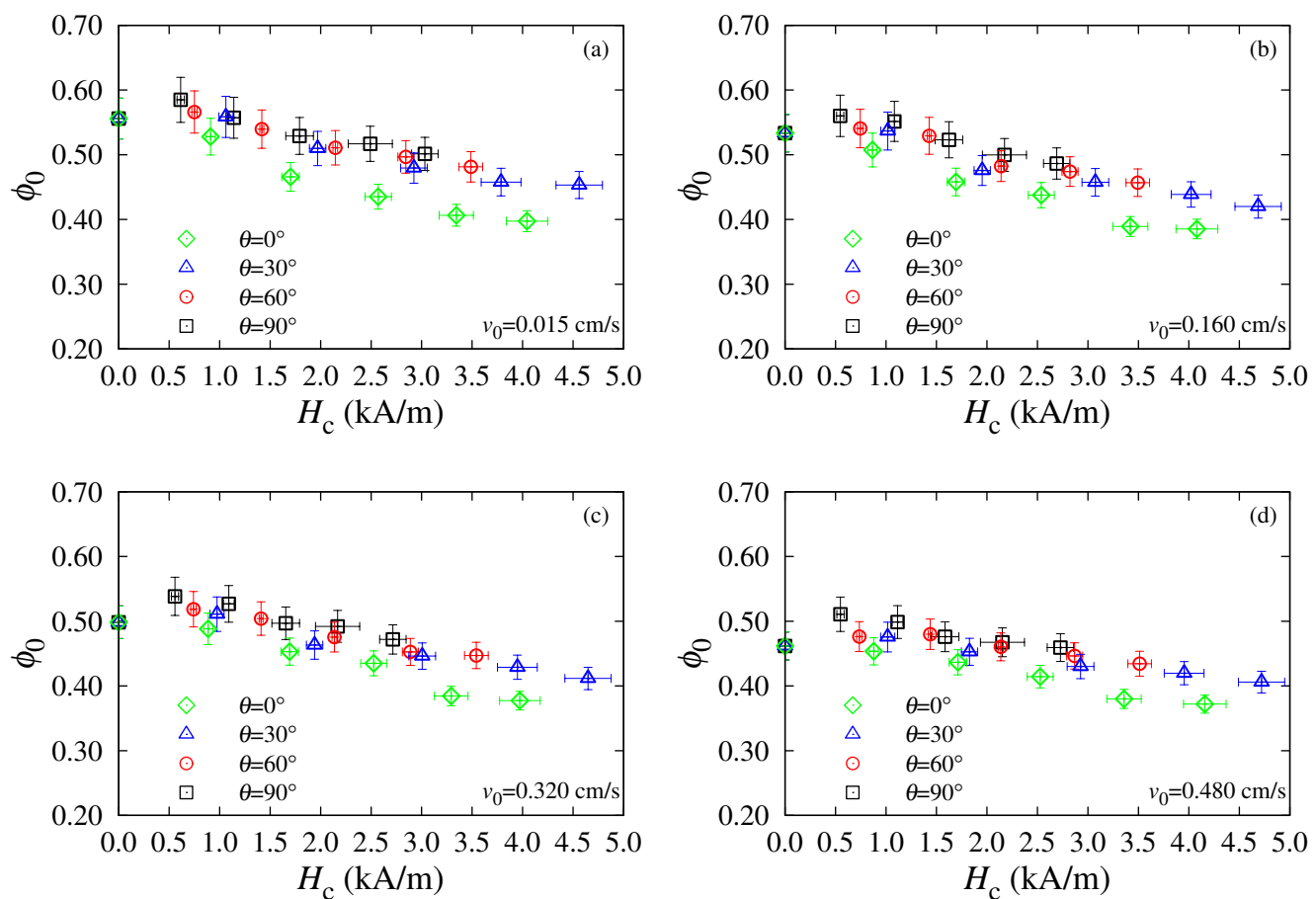

Figure 7: Volume fraction of the powder bed $\phi$ before the breaking point as a function of the intensity $H_{\mathrm{c}}$ and tilted angle $\theta$ of the externally applied magnetic field. Data obtained for $v_{0}=0.015 \mathrm{~cm} / \mathrm{s}(\mathrm{a}), v_{0}=0.160 \mathrm{~cm} / \mathrm{s}(\mathrm{b}), v_{0}=0.320 \mathrm{~cm} / \mathrm{s}(\mathrm{c})$ and $v_{0}=0.480 \mathrm{~cm} / \mathrm{s}(\mathrm{d})$, respectively. 


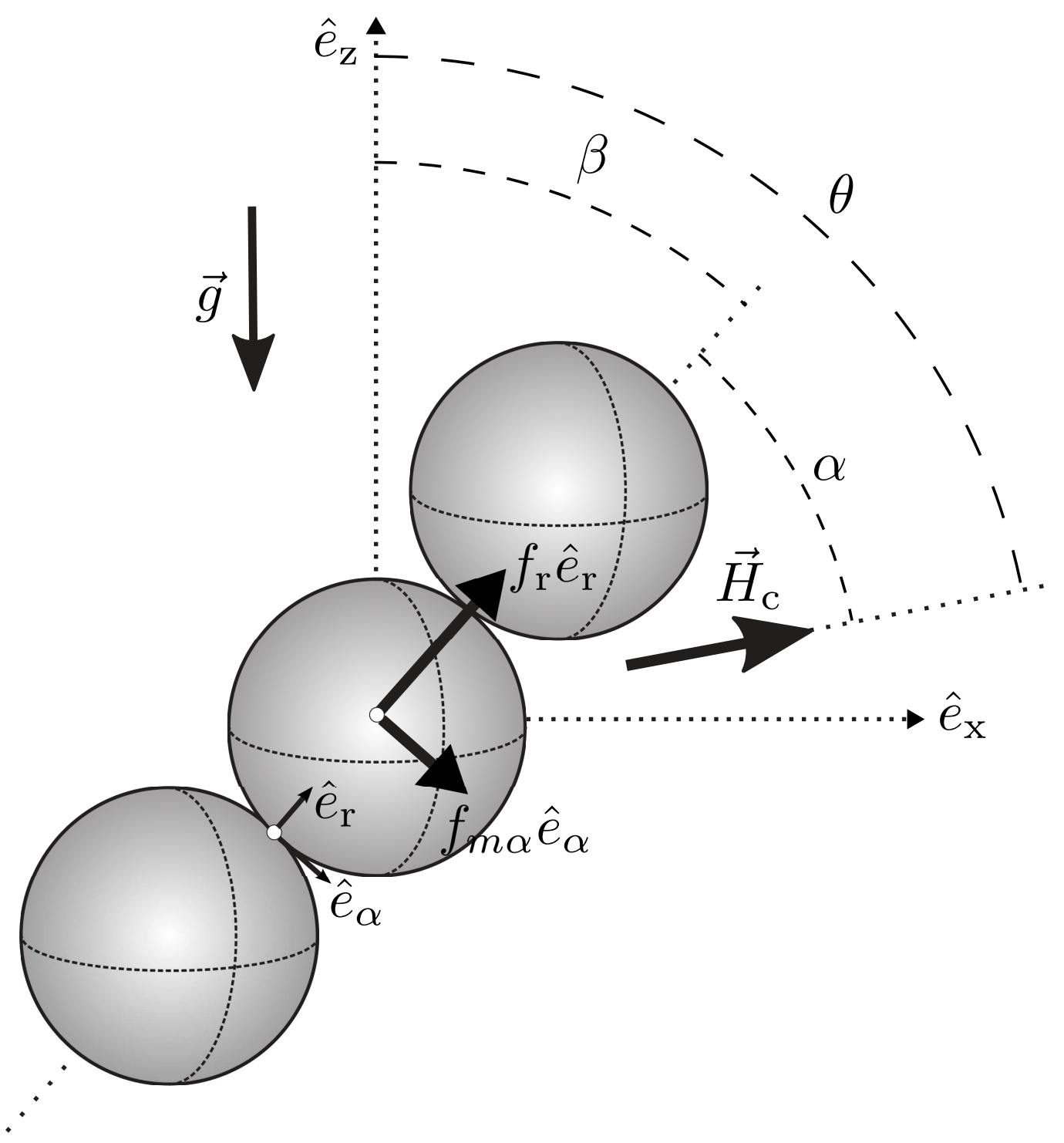

Figure 8: Radial $f_{\mathrm{r}}$ and tangential $f_{m \alpha}$ components of the magnetic force $\vec{f}_{\mathrm{m}}$ between magnetized spheres in the presence of an external magnetic field $\vec{H}_{\mathrm{c}}$ tilted an angle $\theta$ with respect to gravity. The angle between the chained particles and $\vec{H}_{\mathrm{c}}$ is $\alpha$. The angle between the chain of particles and the vertical direction is $\beta$. 


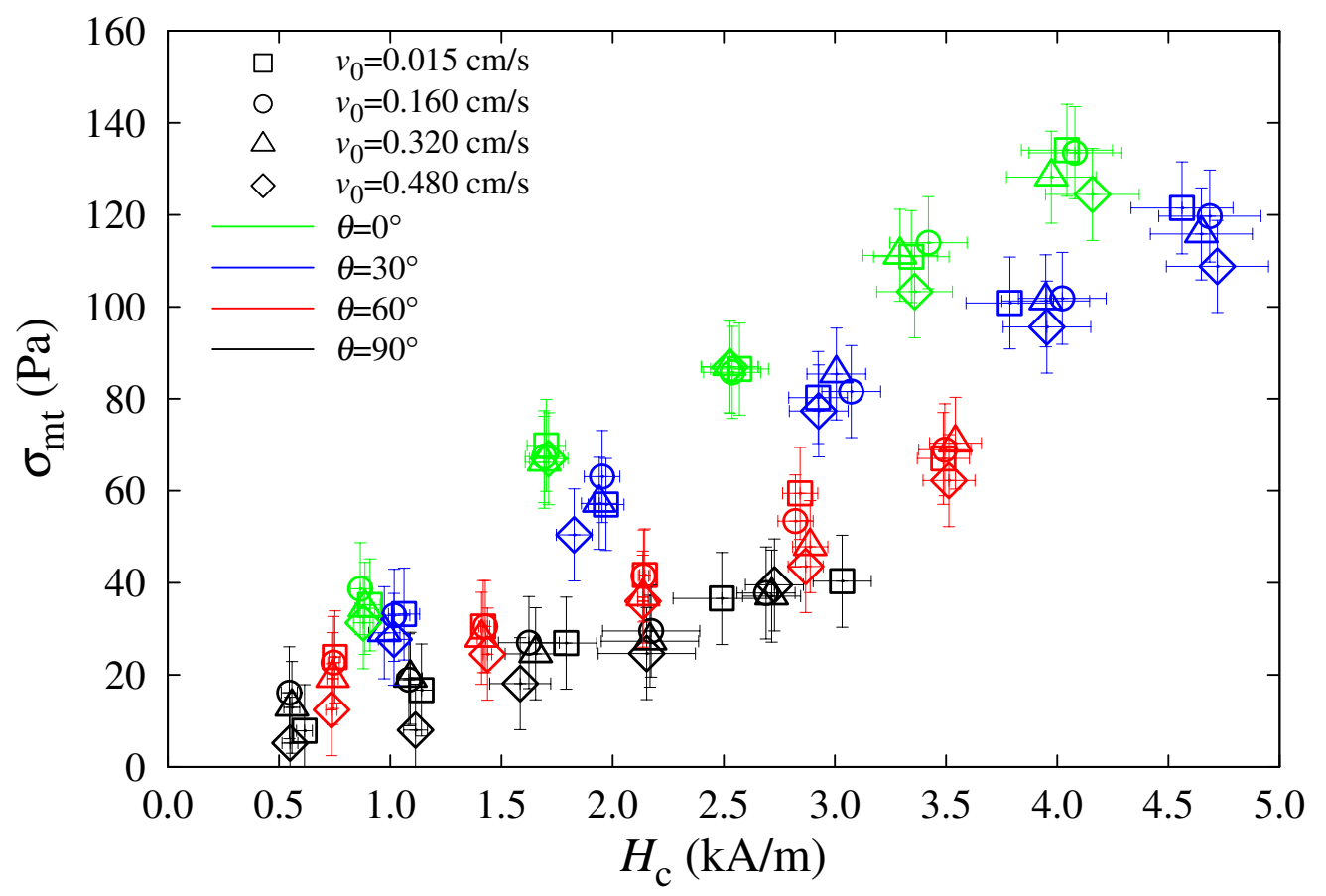

Figure 9: Magnetic yield stress $\sigma_{\mathrm{mt}}$ versus the magnetic field intensity $H_{\mathrm{c}}$ for different values of the tilt angle $\theta$ of the applied field with respect to the vertical direction. Applied consolidation velocities $v_{0}$ are indicated. 

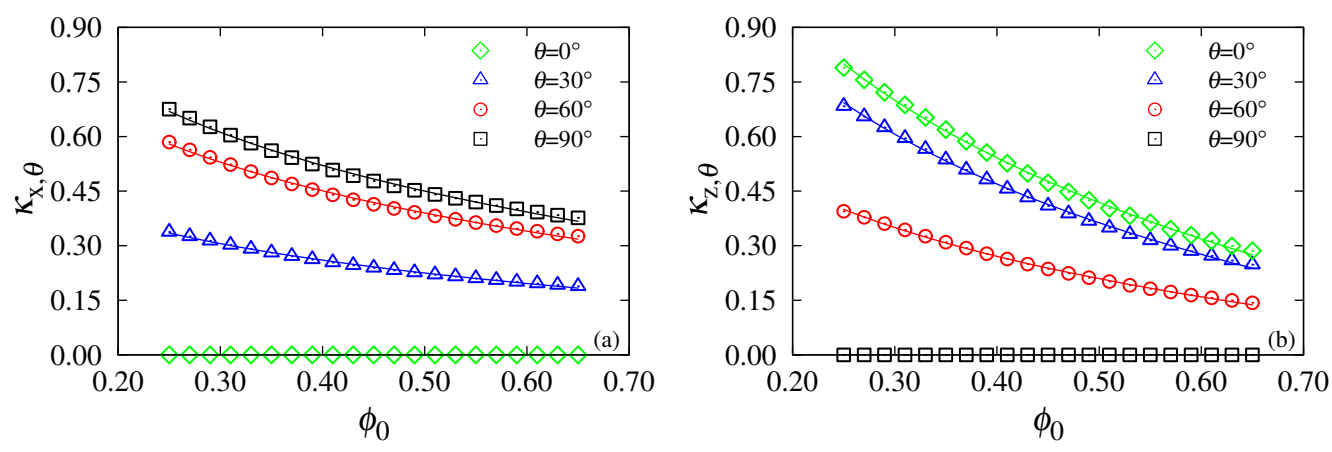

Figure 10: Calculated magnetometric demagnetization factors in the radial (a) and vertical (b) directions for the magnetite powder used in this work as a function of the solid fraction in the bed and the tilt angle $\theta$ of the applied magnetic field. The lines represent the best fits of equation: $\kappa=a-b \ln \phi$. Best fitting parameters $a$ and $b$ are reported in Table 1 .
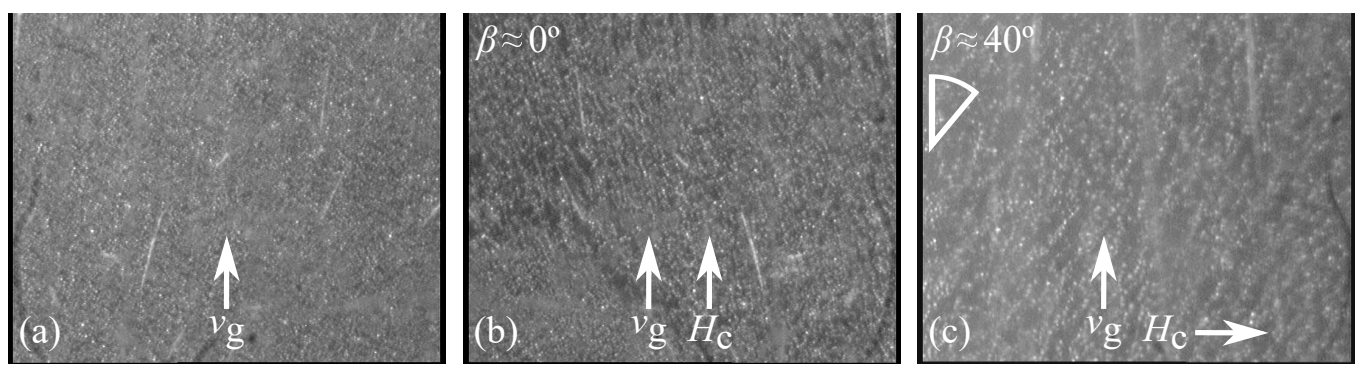

Figure 11: Optical microscope images of the bulk of magnetite MSBs in the absence (a) and presence of a vertically (b) and horizontally (c) orientated magnetic fields. Tilt angle $\beta$ of the chain aggregates are indicated. Samples were stabilized at a consolidation gas velocity $v_{0}=0.015 \mathrm{~cm} / \mathrm{s}(\mathrm{a}-\mathrm{c})$ by a magnetic field of magnitude $H_{\mathrm{c}}=2.85 \mathrm{kA} / \mathrm{m}(\mathrm{b}-\mathrm{c})$. 


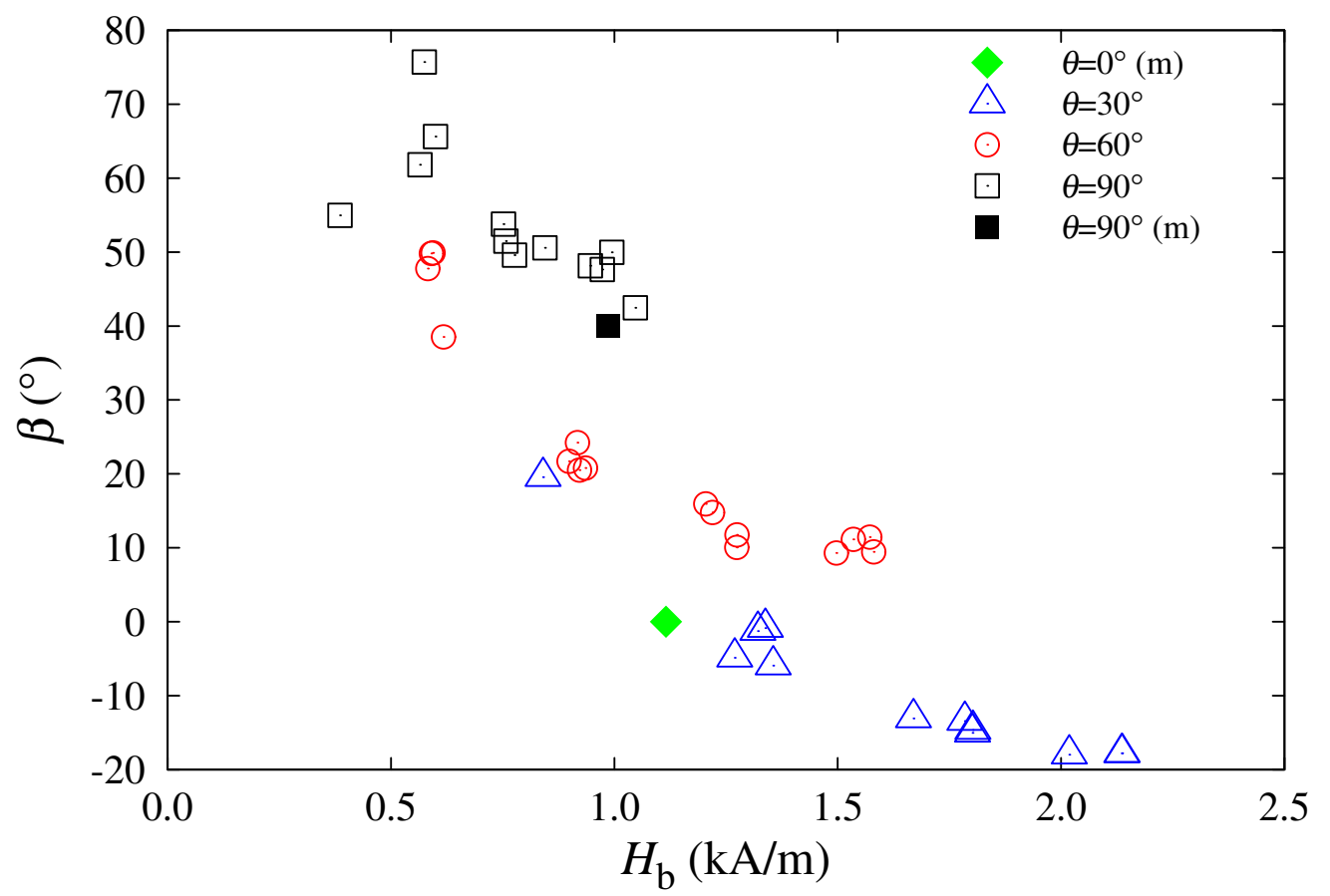

Figure 12: Calculated inclination angle of the chains of particles with respect to the vertical direction as a function of the intensity $H_{\mathrm{c}}$ and tilt angle $\theta$ of the externally applied magnetic field. Measured (m) values of $\beta$ (filled symbols) in Fig. 11 are also shown. 